\title{
Reductions in biomarkers of exposure to selected harmful and potentially harmful constituents following exclusive and partial switching from combustible cigarettes to myblu ${ }^{\text {tm }}$ electronic nicotine delivery systems (ENDS)
}

\author{
Paul Morris ${ }^{1}$ (D) Simon McDermott ${ }^{2} \cdot$ Fiona Chapman $^{2} \cdot$ Thomas Verron $^{2} \cdot$ Xavier Cahours $^{2} \cdot$ Matthew Stevenson $^{2}$. \\ Joseph Thompson ${ }^{2} \cdot$ Nveed Chaudhary ${ }^{2} \cdot$ Grant O'Connell $^{2}$
}

Received: 7 April 2021 / Accepted: 21 July 2021 / Published online: 26 August 2021

(c) The Author(s) 2021

\begin{abstract}
Electronic nicotine delivery systems (ENDS) offer adult combustible cigarette smokers an alternative, potentially reduced harm, mode of nicotine delivery, attributed to fewer and reduced levels of harmful and potentially harmful constituents (HPHCs) in their aerosols compared to cigarette smoke. These two identical, randomised, open label, two-part studies aimed to compare levels of 15 biomarkers of exposure (BoE) to selected HPHCs associated with tobacco smoking in healthy US adult smoker subjects $(n=72)$. Following 9 days of exclusive use of a range of allocated $m y b l{ }^{\text {TM }}$ ENDS variants, subjects' levels of 14 non-nicotine BoE were substantially reduced compared to baseline values (combustible cigarette use), in the range of $46-97 \%$. BoE reductions were sustained in subjects who continued myblu use exclusively $(n=25)$ for a further 5 days, and returned to near baseline levels in subjects who returned to exclusive combustible cigarette use $(n=21)$. Dual users $(n=24)$ demonstrated reductions in BoE to a lesser extent than with exclusive myblu use. Measured nicotine equivalents did not significantly change throughout the study. These data suggest exclusive use of ENDS provides adult smokers seeking an alternative to combustible cigarettes with substantial reductions in HPHC exposures whilst achieving satisfying levels of nicotine delivery. Dual use involving substitution of cigarettes may also provide some of this advantage, but to lesser extent. Overall, the data contribute to the weight of evidence that ENDS are an important tool in tobacco harm reduction for adult smokers unwilling to or uninterested in quitting smoking. Study 1: NCT 04430634, study 2: NCT 04429932, clinicaltrials. gov (10-06-2020).
\end{abstract}

Keywords Tobacco harm reduction · e-Cigarettes · Electronic nicotine delivery systems $\cdot$ Biomarkers of exposure · Smoking $\cdot$ Cigarette

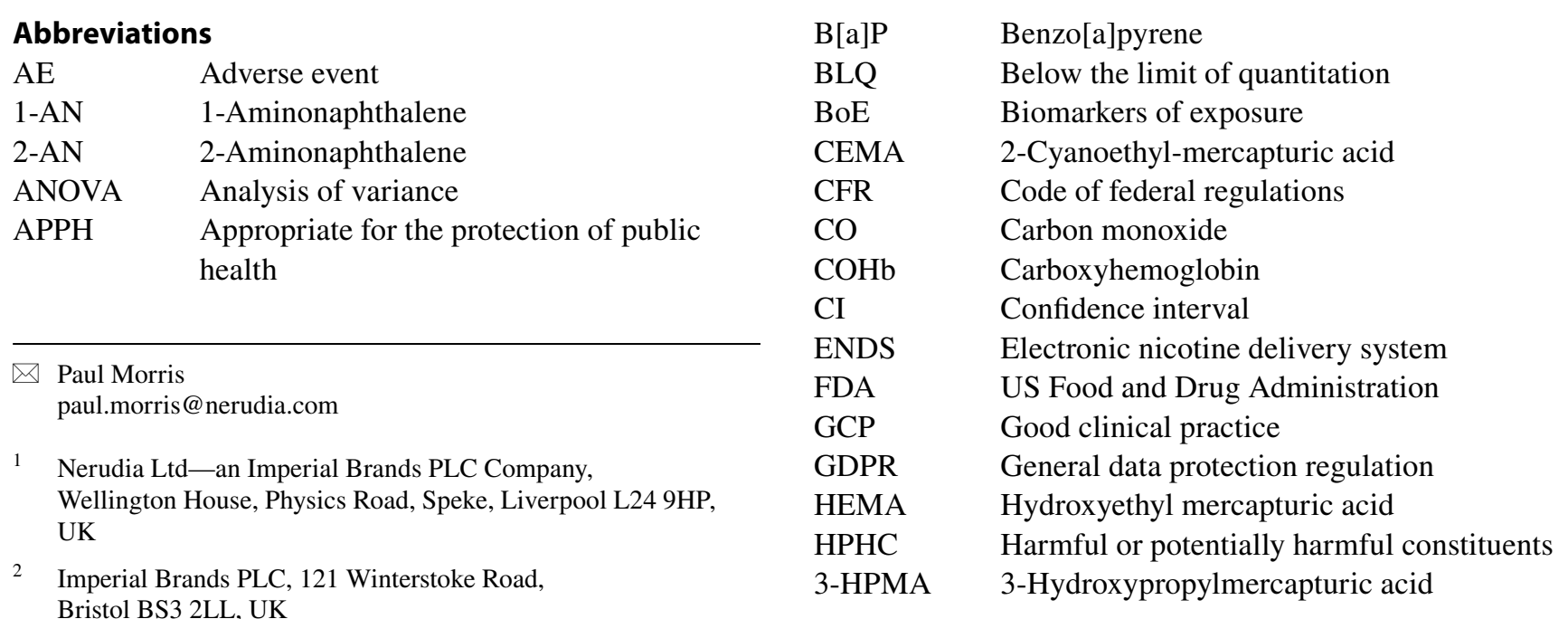




$\begin{array}{ll}\text { 3-HMPMA } & \text { 3-Hydroxy-1-methylpropylmercapturic acid } \\ \text { ICF } & \text { Informed consent form } \\ \text { ICH } & \text { International Council for Harmonisation } \\ \text { IRB } & \text { Institutional Review Board } \\ \text { LS } & \text { Least squares (mean) } \\ \text { MHBMA } & \text { Monohydroxybutenylmercapturic acid } \\ \text { NO } & \text { Nitric oxide } \\ \text { NNAL } & \text { 4-(Methylnitrosamino)-1-(3-pyridyl)-1- } \\ & \text { butanol } \\ \text { NNK } & \text { 4-(Methylnitrosamino)-1-(3-pyridyl)-1- } \\ & \text { butanone } \\ \text { NNN } & \text { N-nitrosonornicotine } \\ \text { 3-OH B[a]P } & \text { 3-Hydroxybenzo[a]pyrene } \\ \text { 1-OHP } & \text { Hydroxypyrene } \\ \text { O-tol } & \text { O-toluidine } \\ \text { PI } & \text { Principal investigator } \\ \text { PK } & \text { Pharmacokinetic } \\ \text { SAE } & \text { Serious adverse event } \\ \text { S-PMA } & \text { S-phenyl mercapturic acid } \\ \text { SD } & \text { Standard deviation } \\ \text { SEM } & \text { Standard error of the mean } \\ \text { THR } & \text { Tobacco harm reduction } \\ \end{array}$

\section{Introduction}

Tobacco harm reduction (THR) involves providing a means by which adult combustible cigarette smokers, who are uninterested or unwilling to quit smoking, can achieve satisfactory nicotine consumption but with exposure to fewer, and substantially reduced levels of, toxicants associated with burning tobacco [1,2]. This is an increasingly important concept and electronic nicotine delivery systems (ENDS) are one such category which may contribute to THR; a growing body of evidence within the scientific literature supports this, along with a number of regulators and public health bodies [1-4]. Recently, a comprehensive Cochrane Review found ENDS are an effective tool in enabling smoking cessation in adult smokers, even among those who do not intend to quit smoking, with no serious unwanted effects or harm with up to 2 years of usage [5]. Clinical data suggest that adult smokers who transition to use of ENDS generally experience improvements in their pulmonary and cardiovascular health $[6,7]$ and there is increasing evidence that the reduced harm potential of ENDS is linked to fewer and/or reduced levels of harmful and potentially harmful constituents (HPHCs) present in their aerosols, compared to smoke generated from cigarette combustion [8-12]. Rudd et al. [8] found that differences in toxicological outcomes in vitro directly correlated with HPHC exposure levels: reduced levels and fewer numbers of HPHCs within ENDS aerosol resulted in substantially reduced cytotoxicity, and negative outcomes for both genotoxicity and mutagenicity, unlike exposure to cigarette smoke. Similar reductions in toxicity of ENDS aerosols when compared to cigarette smoke samples have been recorded in a number of other studies, for example: [13-16].

Further to observations on smoke/aerosol compositions within laboratory settings, a number of clinical studies have also indicated substantially reduced biomarkers of exposure BoE to tobacco smoke HPHCs in adult smokers following exclusive use of ENDS [17-20]. For example, when adult smokers switched to a period of exclusive use of an ENDS device, they experienced substantial reductions in exposure to HPHCs, and in a short amount of time, to levels not dissimilar to those found in adult smokers who had switched to medically licensed nicotine replacement therapies (NRT) or 'cold turkey' cessation [21-23]. In general, previous studies have investigated the effects of older generation ENDS, however, there are fewer studies analysing the $\mathrm{BoE}$ levels associated with newer generation ENDS use; these newer generation products are now a popular alternative for combustible cigarettes used by adult smokers to reduce or replace smoking while still consuming nicotine [24, 25]. Newer generation products commonly take the form of a pod-based system, where a battery-powered segment is combined with a pre-filled 'pod' containing the e-liquid $[8,26]$.

Nicotine can be present in a freebase or nicotine salt form within the e-liquid to satisfy the preference of the adult smoker [27]. Nicotine salt, produced by the reaction of nicotine and a suitable acid, provides improved nicotine delivery to the adult smoker's lung than the more volatile freebase variant and, therefore, results in greater pulmonary absorption, increased blood nicotine levels, and adult smoker satisfaction [28]. A range of flavour/nicotine options has been evidenced to play a role in 'off-ramping' of adult smokers from combustible cigarettes to potentially less harmful forms of nicotine consumption, including ENDS use, by increasing the acceptability and satisfaction of such products based on personal preference $[5,29,30]$. In fact, an important and common part of this off-ramping process is a period of 'dual-use' transition, where adult smokers will continue to use combustible cigarettes but will combine this with ENDS use as they familiarise themselves with these products and gradually reduce the number of combustible cigarettes smoked per day [31]. It has been demonstrated that dual-users are more motivated to quit smoking and are less dependent on combustible cigarette consumption [32]. In fact, there is also some evidence that there is an increased likelihood of dual-users going on to replace cigarette smoking entirely compared to those adult smokers who do not dual use [33]. Additionally, it has been found that adult smokers who become dual-users, and subsequently reduce their combustible cigarette use, experience reduced exposures to combustible tobacco BoE [22, 34]. Therefore, a dual-use arm was included in this study to further investigate 
and quantify the effects of dual use of newer generation ENDS and cigarettes on tobacco smoking BoE.

This study aimed to assess the changes from baseline (Day 1, cigarette smoking) levels of 15 tobacco-smoking related BoE following a 9-day exclusive use period of $m y \mathrm{blu}^{\mathrm{TM}}$ ENDS products. Given some recent societal concerns around the potential additional toxicological burden of e-liquid flavourings, different nicotine strengths and the use of nicotine salts $[35,36]$, we sought to include a range of products (currently available on the US market) to represent these formulaic differences within this study. The second, subsequent, part of this study aimed to assess (in randomised groups) (i) if any changes in BoE levels (compared to baseline) following exclusive myblu use for 9 days were maintained up to 14 days, (ii) the effects on BoE levels (against baseline) following participants' switching back to cigarette smoking between study Days 9 and 14 and (iii) the effects of dual use of cigarettes and myblu ENDS on BoE (against baseline) following participants' switching to this between days 9 and 14 . We selected the $15 \mathrm{BoE}$ [primary: carboxyhemoglobin (COHb; corresponding HPHC, carbon monoxide), 4-(methylnitrosamino)-1-(3-pyridyl)-1-butanol (NNAL; 4-(methylnitrosamino)-1-(3-pyridyl)-1-butanone), 3-hydroxypropyl-mercapturic acid (3-HPMA; acrolein), S-phenyl-mercapturic acid (S-PMA; benzene); secondary: 2-cyanoethylmercapturic acid (CEMA; acrylonitrile), hydroxyethyl mercapturic acid (HEMA; ethylene oxide), 3-hydroxy-1-methylpropyl-mercapturic acid (3-HMPMA; crotonaldehyde), monohydroxybutenyl-mercapturic acid (MHBMA; 1,3-butadiene), o-toluidine (o-tol; toluidine), 1-aminonaphthalene (1-AN; naphthalene), 2-aminonaphthalene (2-AN; naphthalene), N-nitrosonornicotine (NNN; N-nitrosonornicotine), 1-hydroxypyrene (1-OHP; pyrene), 3-hydroxybenzo[a]pyrene (3-OH B[a]P; benzo[a]pyrene), nicotine equivalents (nicotine)] to represent major classes of compounds found within cigarette smoke, the majority of which have been reported by the US FDA to significantly contribute, or potentially contribute, to smoking-related disease risks [37]. This study complements existing pre-clinical toxicological data on the myblu ENDS test samples [8,38] and acts to verify those findings within adult combustible cigarette smoking subjects.

\section{Methods}

\section{Study participants}

Study participants were recruited from areas surrounding the study sites (Celerion, Lincoln, NE; Frontage, Secaucus, $\mathrm{NJ})$ using standard advertising methods and were compensated for their participation in the study. Seventy-two subjects completed the study and met the conditions for inclusion in the data analysis (out of a total of 79 recruited); the study population consisted of healthy US adult smokers aged 21-65 years, who reported smoking ten or more commercially available combustible cigarettes (menthol or non-menthol) per day (for a period of at least 12 months) and did not use any other nicotine-containing products within the 14 days prior to check-in (Day 2). Smoking $>10$ cigarettes per day is a commonly used criterion which represents a typical/moderate level of product usage for regular adult smokers $[39,40]$. As the study aimed to assess the potential of the test products to offer adult smokers an alternative mode of nicotine delivery with THR potential due to reduced exposure to HPHCs, exclusive smoker adults who were prepared to switch for the duration of the study but were otherwise not intending to alter their smoking habits were recruited for this study. The participants self-reported that they had not used other nicotine-containing products for 14 days prior to the study, and this criterion was applied to remove any confounding effects on measurements from such products' use. However, ENDS or other nicotine product use prior to this 14 day period were not restricted. Smoking status was confirmed via urine cotinine $(\geq 200 \mathrm{ng} / \mathrm{mL})$ and exhaled carbon monoxide ( $>10 \mathrm{ppm}$ ) levels [41].

Study exclusion criteria included a history or presence of clinically significant mental or physical health conditions; females who were pregnant or breastfeeding; high blood pressure (vital sign ranges used at screening were based on the clinic sites' standard ranges: systolic blood pressure 90-150 $\mathrm{mmHg}$, diastolic blood pressure $40-95 \mathrm{mmHg}$ and heart rate $40-99 \mathrm{bpm}$ ); body mass index $<18 \mathrm{~kg} / \mathrm{m}^{2}$ or $>40 \mathrm{~kg} / \mathrm{m}^{2}$; acute illnesses (e.g., upper respiratory infection, viral infection) requiring treatment within 14 days prior to check-in; use of prescription smoking cessation treatments, anti-diabetic or insulin drugs or medications known to interact with Cytochrome P450 2A6; positive urine screen for alcohol or drugs of abuse; and self-reported puffers or mouth-hold smokers (i.e., smokers who draw smoke from a combustible cigarette into the mouth and throat but do not inhale).

The studies were carried out in accordance with: title 21 of the Code of Federal Regulations (21 CFR) parts 50, 56, 312 ; the US FDA electronic nicotine delivery systems guidance for industry [42] for biomarker endpoint assessment; International Council on Harmonisation (ICH) guidelines regarding Good Clinical Practice (E6 Consolidated Guidance, April 1996) [43]; the ethical principles set forth in the Declaration of Helsinki; all other relevant laws including those in relation to the protection of subjects data under the General Data Protection Regulation (GDPR); the approved study protocols. The study protocol for study 1 was reviewed and approved by Advarra Institutional Review Board (IRB) (Columbia, MD) and the study protocol for Study 2 was reviewed and approved by IntegReview IRB (Austin, TX). 
Any subsequent amendments were also approved by the same respective IRBs. Both the Advarra IRB and IntegReview IRB were constituted and operated in accordance with the principles and requirements described in the $21 \mathrm{CFR}$ Part 56; both IRBs are compliant to ICH guidelines. All subjects provided informed consent. All prospective subjects had the study explained to them by the Investigator or their designee and were required to read, sign and date an Institutional Review Board (IRB) approved Informed Consent Form (ICF) prior to completion of screening or other study procedures. This consent form provided the subjects in nontechnical terms with the purpose of the study, procedures to be carried out, and potential hazards. The subjects were assured that they may withdraw from the study at any time without jeopardising medical care related to or required as a result of study participation. Subjects were given a copy of their signed ICF. Study 1 (CA22749) was registered at www.clinicaltrials.gov with ID: NCT 04430634. Study 2 (CA22736) study was registered at www.clinicaltrials.gov with ID: NCT 04429932.

\section{Products}

The ENDS test products used in this study were the commercially available $m y b l{ }^{\mathrm{TM}}$ two-piece closed system comprised of a rechargeable $350 \mathrm{mAh}$ battery and disposable pod containing an e-liquid. The e-liquid mixtures consisted of VG, PG, nicotine and a proprietary blend of flavours; pods contained $1.5 \mathrm{~mL}$ of e-liquid, equating to approximately 200 puffs under standardised machine puffing conditions [28]. Sixteen commercial disposable liquid pod variants were included in this study (Table 1). The pods also include the mouthpiece and heating element, and connect directly to, and are only compatible with, the myblu ENDS battery section. During use, the consumer inhaled through the mouthpiece and a sensor in the battery section detected the resulting change in air pressure, and in turn the heating element in the pod was activated, heating the e-liquid to an aerosol subsequently inhaled by the user. An example image of the product can be found in the supplementary information (Fig. 4).

The ENDS were charged and assembled for the participants and product information sheets provided. On each Study Day, fresh pods and a fully charged device were provided. All participants received training from clinic staff on how to operate their ENDS and to ensure compliance in the clinic, all participants used their products under the supervision of suitably qualified staff.

\section{Study design and procedure}

The study was conducted under the oversight of Celerion in two US-based clinical research centres (Celerion, Lincoln,
Table 1 Details of the myblu ENDS product variants used in Studies 1 and 2

\begin{tabular}{llll}
\hline $\begin{array}{l}\text { Product } \\
\text { identi- } \\
\text { fier }\end{array}$ & Product variant & $\begin{array}{l}\text { Nicotine } \\
\text { strength } \\
(\mathrm{w} / \mathrm{v})\end{array}$ & Nicotine form \\
\hline $\begin{array}{l}\text { Study 1 } \\
\text { 1A }\end{array}$ & Intense tobacco (2.4\%) & $24 \mathrm{mg} / \mathrm{mL}$ & Nicotine salt \\
1B & Intense melon mint & $36 \mathrm{mg} / \mathrm{mL}$ & Nicotine salt \\
& $(3.6 \%)$ & & \\
1C & Intense fresh melon & $25 \mathrm{mg} / \mathrm{mL}$ & Nicotine salt \\
& $(2.5 \%)$ & & \\
1D & Intense tangerine cream & $40 \mathrm{mg} / \mathrm{mL}$ & Nicotine salt \\
& $(4.0 \%)$ & & \\
1E & Intense tobacco (3.6\%) & $36 \mathrm{mg} / \mathrm{mL}$ & Nicotine salt \\
1F & Intense melon mint & $24 \mathrm{mg} / \mathrm{mL}$ & Nicotine salt \\
& $(2.4 \%)$ & & \\
1G & Intense fresh melon & $40 \mathrm{mg} / \mathrm{mL}$ & Nicotine salt \\
& $(4.0 \%)$ & & \\
1H & Intense fresh mint (3.6\%) & $36 \mathrm{mg} / \mathrm{mL}$ & Nicotine salt \\
Study 2 & & & \\
2A & Gold leaf (2.4\%) & $24 \mathrm{mg} / \mathrm{mL}$ & Freebase nicotine \\
2B & Polar mint $(2.4 \%)$ & $24 \mathrm{mg} / \mathrm{mL}$ & Freebase nicotine \\
2C & Cherry (2.4\%) & $24 \mathrm{mg} / \mathrm{mL}$ & Freebase nicotine \\
2D & Vanilla (2.4\%) & $24 \mathrm{mg} / \mathrm{mL}$ & Freebase nicotine \\
2E & Gold leaf $(1.2 \%)$ & $12 \mathrm{mg} / \mathrm{mL}$ & Freebase nicotine \\
2F & Polar mint $(1.2 \%)$ & $12 \mathrm{mg} / \mathrm{mL}$ & Freebase nicotine \\
2G & Menthol (2.4\%) & $24 \mathrm{mg} / \mathrm{mL}$ & Freebase nicotine \\
2H & Intense fresh mint $(2.4 \%)$ & $24 \mathrm{mg} / \mathrm{mL}$ & Nicotine salt \\
\hline & & & \\
\hline
\end{tabular}

Products were assigned codes $\mathrm{A}-\mathrm{H}$ for randomisation of product use during Part 1 of the study

NE; Frontage, Secaucus, NJ). Two identical studies were split between the two locations to enable recruitment of the required numbers of participants (based on subject availability on volunteer databases at the time of the study) and according to clinical space available at the time of the study. Subjects were enrolled to participate in a randomised, open label, two-part study. All subjects participating in the study were expected to participate in both study parts, with Part 2 continuing immediately on from Part 1 . Subjects were confined to the respective clinics for the full duration of the study. The objectives of the study were the measurement of change-from-baseline differences in combustible cigarette smoking-related BoE following 9 days of exclusive use of a variety of myblu ENDS products (Part 1), then to assess the differences in change from baseline following randomisation of participants to (I) exclusive use of allocated myblu ENDS products, (J) exclusive combustible cigarette smoking or (K) dual use of combustible cigarettes and myblu ENDS products between days 9 and 14 (Part 2). Part 1 of the study was over 9 days to align with data collection for a pharmacokinetic (PK) study (data not presented here); the 9 day period was also selected as an appropriate timeframe to observe 


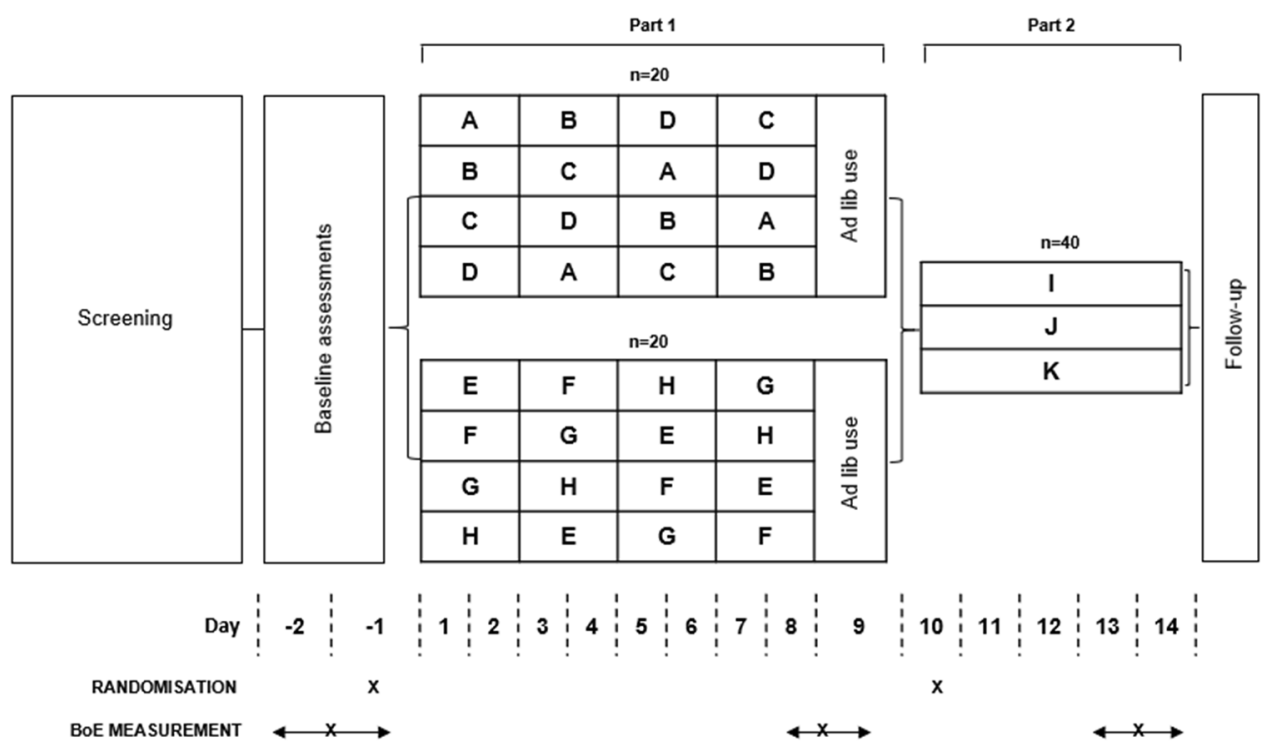

Fig. 1 Overview of the study design. Following screening, participants checked-in to their respective clinical research unit on Day 2. On Days 2 through $1(24 \mathrm{~h})$ baseline BoE assessments were made followed by randomisation to the myblu ENDS product use sequences detailed, Days 1 through 9 (Part 1). Details of the products $($ A-H) for identical Studies 1 and 2 can be found in Table $1(n=$ number of par-

reductions in BoE, as seen with previous studies over shorter time periods [17, 28]. The 5 day period selected for Part 2 of the study was deemed sufficient for the detection of changes in biomarkers [17] following switching of participants to their respective product use arms.

Participant screening procedures were performed within 28 days prior to study procedures and those who successfully completed these and met the eligibility criteria were invited to check-in to their respective clinical research unit on Day 2. At this point the participants' eligibility was re-confirmed, then brief (approximately $30 \mathrm{~min}$ ) training was undertaken on using the $m y$ blu ENDS device with a $0 \%$ nicotine e-liquid to enable the participants to familiarise themselves with use of the device. Baseline (participants using their usual brand of combustible cigarettes) assessments (blood and urine collection) were performed on Days-2 through -1. Subjects were required to abstain from use of any tobacco- or nicotine-containing products for at least $12 \mathrm{~h}$ prior to the start of product use on Day 1. A $12 \mathrm{~h}$ period was used as this equates to approximately $6 \times$ the half-life of nicotine in human subjects [44] and was deemed a sufficient time period to ensure residual nicotine washout from the subjects' systems prior to the start of daily procedures in the clinic. This was carried out overnight to prevent impacting on the subjects' natural product usage behaviour. On Day 1, subjects were randomised to one of eight product use sequences (Fig. 1).

In Part 1 (Days 1-9) of each Study, 20 (intended number) participants were to be randomised to use products ticipants intended to participate in one Study). In Part 2 of the Studies, participants were randomised to one of three study arms (I, J or $\mathrm{K}$, detailed in Table 2). Participant follow-up was carried out approximately 14 days after the end of the study to determine if any adverse events had occurred

A-D and 20 (intended number) subjects randomised to use products E-H from their respective Study (Table 1) (total 80 intended participants in the two Studies combined). On Days 1, 3, 5 and 7, subjects were assigned a myblu device and study product for exclusive use for 2 days, according to the randomisation scheme (Fig. 1). On Days 2, 4, 6 and 8 , participants took part in controlled product use sessions (i.e., 10 puffs taken at $30 \mathrm{~s}$ intervals, $3 \mathrm{~s}$ puff duration) for a pharmacokinetic (PK) data not reported in the current study. Following these controlled use sessions, subjects were instructed to continue using their assigned study product ad libitum for the rest of the day until the start of the next overnight abstinence period. On Day 9, participants were given the option to use any or a mixture of the four products they had been assigned (Days 1-8) in an ad libitum manner until 23:00. Urine samples for BoE assessment were collected over 24 h (Days 8-9); blood was collected on Day 9.

Part 2 of the study commenced on the morning of Day 10 and ran to the end of the study on Day 14. The participants recruited to each study (Study 1: $n=37$; Study 2: $n=35$ ) were randomised to one of three study groups (arms): I (Study 1: $n=14$; Study 2: $n=11$ ), J (Study 1: $n=11$; Study 2: $n=10$ ) and K (Study 1: $n=12$; Study 2: $n=12$ ), detailed in Table 2. Note, two participants from Part 1 were not included in the randomisation to Part 2 as they withdrew consent at this point for personal reasons.

Subjects randomised to exclusive use myblu ENDS products (I) and the dual-use arm (K) had the option to use any 
Table 2 Arms of Part 2 of the study, to which participants were randomised following Part 1

\begin{tabular}{lll}
\hline Study arm & Products & Description \\
\hline $\mathrm{I}$ & myblu ENDS & Exclusive use of myblu ENDS products (A-H) ad libitum \\
$\mathrm{J}$ & Combustible cigarettes & $\begin{array}{l}\text { Exclusive smoking of usual brand combustible cigarettes ad libitum } \\
\text { K }\end{array}$ \\
$\begin{array}{l}\text { Dual use } \\
\text { (myblu ENDS/combus- } \\
\text { tible cigarette) }\end{array}$ & $\begin{array}{c}\text { Use of usual brand combustible cigarettes up to 50\% of the } \\
\text { subject's self-reported use and of } m y b l u \text { ENDS products (A-H) } \\
\text { ad libitum }\end{array}$ \\
\hline
\end{tabular}

Details of products A-H for both Studies 1 and 2 can be found in Table 1 of the eight study products $(\mathrm{A}-\mathrm{H})$ available in Part 1 of their respective Study (Study 1 or 2). Urine samples were collected over $24 \mathrm{~h}$ [Days 13 (after the end of Day's product use) -14]; blood was collected on Day 14. Product use behaviour was assessed by documenting the number of cigarettes smoked, the flavours and strength of the myblu ENDS products and pod masses, per day as appropriate (data not shown).

The clinical research units attempted to contact all subjects who used at least one study product (including subjects who terminated the study early), using their standard procedures, approximately 14 days after the last study product use to determine if any adverse events (AEs) had occurred since their last study visit.

\section{Product use}

Use of the assigned products was documented daily and subjects were monitored during clinical confinement to ensure that no illicit nicotine or tobacco products were used. All product use was ad libitum from 07:30 to 23:00 each day apart from on days 2, 4, 6 and 8, on which controlled use sessions were held for additional PK studies (data not shown here). Other exceptions included during meals, overnight abstinences prior to the PK profiling periods, 15 min prior to blood sampling, and 30 min prior to exhaled carbon monoxide (CO) and nitric oxide (NO) measurements. Subjects were not permitted to use any nicotine or tobacco products other than those allocated during the study and were not permitted to remove any study products from their clinic facility.

In the second part of the study, subjects randomised to the dual-use groups were required to request conventional tobacco cigarette products from the clinic staff and smoke only in specified sections of the clinic, away from non-smoking subjects. To standardise cigarette consumption during the study, subjects in the dual-use groups were required to reduce their daily cigarette consumption by $\sim 50 \%$ that reported at screening. Cigarette consumption data (Days 10-14) can be found in Tables 10 and 12 of the supplementary information.

Subjects randomised to receive the myblu ENDS products were able to carry them throughout the day, within designated sections of the clinic. New myblu ENDS products were supplied to the subjects each morning and throughout the day if the e-liquid solution was fully consumed, if the product failed to work properly, or if the subject requested an alternative flavour (from within their assigned randomisation). All myblu ENDS products were weighed before and after use to provide a consumption estimate (Tables 11 and 13 , supplementary information).

\section{Determination of sample size}

The selected intended sample size $(n=80)$ was deemed adequate to meet the study objectives, based on previous clinical assessment with myblu ENDS products [22]. In a similar BoE assessment using myblu products, O'Connell et al. [22] estimated that a sample size of 12 was required to detect a $70 \%$ reduction from baseline in participant groups that stopped smoking for the study, and differences between groups could be detected with at least $80 \%$ power in twosided analyses. Their study assigned 15 subjects to each group to maximise the likelihood of a minimum of 12 subjects per group completing the study. Therefore, we intended to allocate the participants of each Study to their respective arms in a ratio of 14:13:13 I:J:K.

\section{Biomarkers of exposure analyses}

The urine and blood BoE measured in this study were selected to represent major classes of HPHCs that have previously been reported for conventional tobacco cigarette smokers [45-50] and are detailed in Table 3. Blood samples for measurement of whole blood $\mathrm{COHb}$ were collected in the afternoon of Days-1, 9 and 14. $24 \mathrm{~h}$ urine collections were carried out for measurement of NNAL, 3-HPMA, S-PMA, CEMA, HEMA, 3-HMPMA, MHBMA, o-tol, 1-AN, 2-AN, NNN, 1-OHP, 3-OH B [a]P and nicotine equivalents on Days 2-1, 8-9 and 13-14. Each $24 \mathrm{~h}$ urine collection commenced at the start of overnight abstinence. Measurement of each biomarker was carried out using validated methods based on: FDA's Guidance to Industry for Bioanalytical Method Validation [51]; Good Laboratory Practices per 21 CFR Part 58 [52]; and the EMEA Guideline on Bioanalytical Method Validation (EMEA/CHMP/EWP/192217/2009 Rev. 1 Corr.2) [53]. 
Table 3 Biomarkers of exposure (BoE) used in this study and the associated harmful or potentially harmful constituents (HPHC) (present in cigarette smoke)

\begin{tabular}{|c|c|c|c|c|c|}
\hline \multicolumn{3}{|l|}{$\mathrm{BoE}$} & \multirow[t]{2}{*}{ HPHC } & \multirow[t]{2}{*}{ HPHC category } & \multirow{2}{*}{$\begin{array}{l}\text { Associated } \\
\text { health risk }\end{array}$} \\
\hline Chemical name & Abbreviation & Matrix & & & \\
\hline Carboxyhemoglobin & $\mathrm{COHb}$ & Blood & Carbon monoxide (CO) & Carbon oxides & RDT \\
\hline 4-(Methylnitrosamino)-1-(3-pyridyl)-1-butanol & NNAL & Urine & $\begin{array}{l}\text { 4-(Methylnitrosamino)-1-(3- } \\
\text { pyridyl)-1-butanone } \\
\text { (NNK) }\end{array}$ & TSNA & $\mathrm{CA}$ \\
\hline 3-Hydroxypropyl-mercapturic acid & 3-HPMA & Urine & Acrolein & VOC & $\begin{array}{l}\text { RT } \\
\text { CT }\end{array}$ \\
\hline S-Phenyl-mercapturic acid & S-PMA & Urine & Benzene & VOC & $\begin{array}{l}\text { CA } \\
\text { CT } \\
\text { RDT }\end{array}$ \\
\hline 2-Cyanoethylmercapturic acid & CEMA & Urine & Acrylonitrile & VOC & $\begin{array}{l}\text { CA } \\
\text { RT }\end{array}$ \\
\hline Hydroxyethyl mercapturic acid & HEMA & Urine & Ethylene oxide & VOC & $\begin{array}{l}\text { CA } \\
\text { RT } \\
\text { RDT }\end{array}$ \\
\hline 3-Hydroxy-1-methylpropyl-mercapturic acid & 3-HМPMA & Urine & Crotonaldehyde & VOC & $\mathrm{CA}$ \\
\hline Monohydroxylbutenyl-mercapturic acid & MHBMA & Urine & 1,3-Butadiene & VOC & $\begin{array}{l}\text { CA } \\
\text { RT } \\
\text { RDT }\end{array}$ \\
\hline o-Toluidine & o-tol & Urine & Toluidine & Aromatic amines & CA \\
\hline 1-Aminonaphthalene & $1-\mathrm{AN}$ & Urine & Naphthalene & Aromatic amines & $\begin{array}{l}\text { CA } \\
\text { RT }\end{array}$ \\
\hline 2-Aminonaphthalene & 2-AN & Urine & Naphthalene & Aromatic amines & $\begin{array}{l}\text { CA } \\
\text { RT }\end{array}$ \\
\hline $\mathrm{N}$-Nitrosonornicotine & $\mathrm{NNN}$ & Urine & $\mathrm{N}$-nitrosonornicotine $(\mathrm{NNN})$ & TSNA & CA \\
\hline 1-Hydroxypyrene & 1-OHP & Urine & Pyrene & PAH & N/A \\
\hline 3-hydroxybenzo[a]pyrene & 3-OH B[a]P & Urine & Benzo[a]pyrene (B[a]P) & PAH & $\mathrm{CA}$ \\
\hline Nicotine equivalents & Nicotine equivalents & Urine & Nicotine & Nicotine & $\begin{array}{l}\text { RDT } \\
\mathrm{AD}\end{array}$ \\
\hline
\end{tabular}

Pyrene is not classified by the FDA but is used as a marker for PAH (including B[a]P) exposure [50]

TSNA tobacco specific nitrosamine, VOC volatile organic compound, $P A H$ polycyclic aromatic hydrocarbon, RDT reproductive or developmental toxicant, $C A$ carcinogen, $R T$ respiratory toxicant, $C T$ cardiovascular toxicant, $A D$ addictive as classified by the FDA [37]\}

\section{Data and statistical evaluation}

For each urine biomarker, the measured concentration, total biomarker mass excreted per $24 \mathrm{~h}$ and creatinine adjusted levels were determined. From these, absolute and percent change from baseline were determined for the mass excreted and creatinine adjusted values. The total urine biomarker mass excreted per $24 \mathrm{~h}$ change-from-baseline values were used for the statistical analyses. BoE concentrations reported as below the limit of quantification (BLQ) were reported as such but were set to one half of the limit of quantification for the purpose of data analyses. Statistical Analysis Software (SAS) was used for all data summarisations and statistical analyses. Data were listed by subject, sequence, and study day and summarised by study day (Day 1 and Day 9) in Part 1, by study day (Day 1 and Day 14) in Part 2 for arms I only, and by study arm and study day (Day 9 and Day 14) in Part 2. Absolute and percent change-from-baseline values were also listed and summarised. Descriptive statistics \{ number of observations [N], mean, median, standard deviation (SD), standard error of the mean (SEM), coefficient of variation (CV; \%), minimum, and maximum) were presented; all data summarisations and figures were generated using the Outcomes Population. Using SAS PROC MIXED, comparisons of the Day 9 and Day 1, and Day 14 and Day 1 in the I arms, primary and secondary BoE values were made using a linear mixed model analysis of variance (ANOVA). The ANOVA model included day as a fixed effect and subject as a random effect. Least square (LS) mean values were detailed for each day. LS mean differences, 95\% CIs of LS mean differences and $p$ values are detailed for the day comparisons. Analyses among the different study arms within the two respective Studies were also carried out as described above, with the arm as the fixed effect and subject as the random effect, and LS means detailed for each arm. 


\section{Results}

For both Studies 1 and 2, comparisons were made between the measured BoE on Day 1 (baseline; cigarette consumption) and Days 9 or 14 for the exclusive myblu use arms (1I and 2I) (Tables 4 and 5, Supplementary information). All BoE, except for nicotine equivalents, demonstrated statistically significant reductions from baseline levels at Days 9 and 14. In Study 1, decreases in the primary $\mathrm{BoE}(\mathrm{COHb}$, NNAL, 3-HPMA and S-PMA) from baseline on both Days 9 and 14 ranged from 72 to $97 \%$; in Study 2, this decrease range was 69 to $93 \%$. Similar decreases were also observed for the secondary BoE (mercapturic acid metabolite BoE (CEMA, HEMA, 3-HMPMA, and MHBMA), aromatic amine BoE (o-tol, 1-AN, 2,-AN), 1-OHP, 3-OH B[a]P and NNN), with the range of reduction across Days 9-14 between 70 and $97 \%$ for Study 1 and 45 and $97 \%$ for Study 2. No statistically significant differences in nicotine equivalent levels compared to baseline were observed in either Study on Days 9 or 14. This was expected as subjects were using their products ad libitum to achieve their preferred level of nicotine satisfaction. Figures 2, 3 detail the levels of 14 BoE (all those measured except nicotine equivalents) at Days 9 and 14 for all the three arms of Studies 1 and 2, respectively, expressed as percentage reduction compared to the relevant baseline levels. More details on the results can be found in the Supplementary information (Tables 4, 5).

Statistical analysis was also carried out between the three product use arms of the respective Studies (Tables 6, 7, Supplementary information). No significant differences were found between the NNN and nicotine equivalent BoE in all three product use arms of Study 1 on comparison of the differences between Days 9 and 14 (part 2 of the study). While no differences were observed between NNAL and HEMA BoE levels in the myblu (1I) and dual-use (1 K) arms of Study 1 on comparison of changes between Days 9 and 14 , their levels in the combustible cigarette arm $(1 \mathrm{~J})$ were significantly different to these two other respective arms. All of the remaining BoE in Study 1 were demonstrated to be

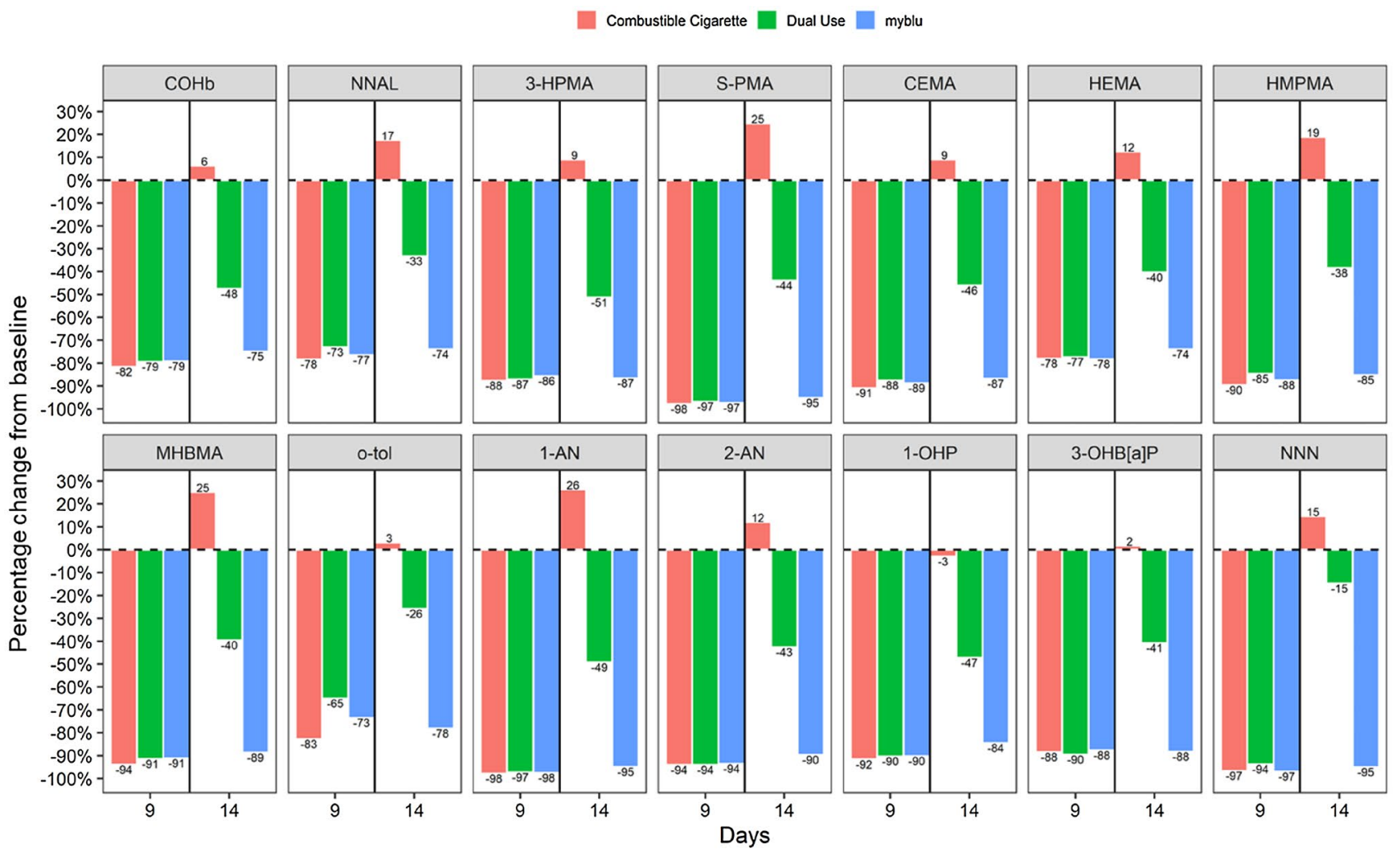

Fig. 2 Relative levels of 14 biomarkers of exposure (BoE) following selected product use, measured at Days 9 and 14 of Study 1. Values are expressed relative (\%) to baseline levels (combustible cigarette smoking) measured on Day 1 and are detailed in labels on the bars. Red bars represent participants who switched from exclusive myblu use to exclusively smoking their usual brand of cigarette on Day 10 of the Study (arm $1 \mathrm{~J})(n=11)$; green bars represent dual-use $(50 \%$ reported usual brand cigarette smoking and myblu use ad libitum from Day 10 of the Study) participants (arm $1 \mathrm{~K})(n=12)$; blue bars represent participants who continued to exclusively using their selected myblu products ad libitum from Day 10 of the Study (arm 1I) $(n=14)$. Definitions of abbreviated BoE can be found in Table 3 (color figure online) 


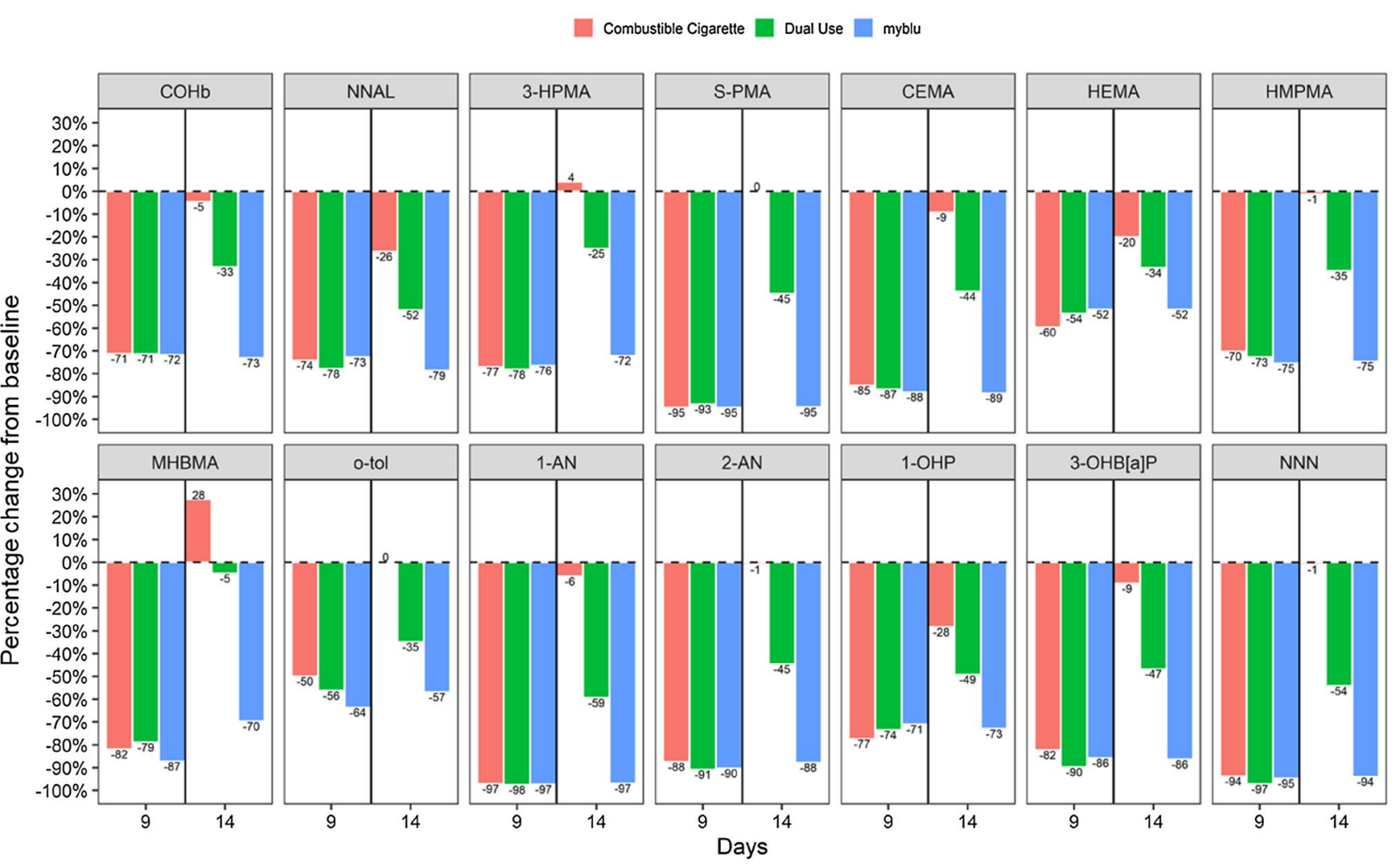

Fig. 3 Relative levels of 14 biomarkers of exposure following selected product use, measured at Days 9 and 14 of Study 2. Values are expressed relative (\%) to baseline levels (combustible cigarette smoking) measured on Day 1 and are detailed in labels on the bars. Red bars represent participants who switched from exclusive myblu use to exclusively smoking their usual brand of cigarette on Day 10 of the Study $(\operatorname{arm} 2 \mathrm{~J})(n=10)$; green bars represent dual-use $(50 \%$

significantly different between the three product use arms on comparison of changes between Days 9 and 14. In Study 2, significant differences between the nicotine equivalent $\mathrm{BoE}$ across Days 9-14 were observed between the combustible cigarette arm $(2 \mathrm{~J})$ and dual-use arm $(2 \mathrm{~K})$. However, no differences were observed between the myblu arm (2I) and either $2 \mathrm{~J}$ or $2 \mathrm{~K}$. On comparison of $2 \mathrm{~J}$ and $2 \mathrm{~K}$, there were no significant differences between changes in NNAL and HEMA levels between Days 9 and 14. However, significant differences were observed between $2 \mathrm{I}$ and $2 \mathrm{~J}$ and between $2 \mathrm{I}$ and $2 \mathrm{~K}$ for these two BoE. In contrast, no differences in changes between Days 9 and 14 were observed for MHBMA, o-tol and NNN on comparison of $2 \mathrm{I}$ and $2 \mathrm{~K}$, whereas differences between $2 \mathrm{I}$ and $2 \mathrm{~J}$, and $2 \mathrm{~J}$ and $2 \mathrm{~K}$, were statistically significant. All the remaining BoE in Study 2 were found to be significantly different between the three product use arms on comparison of changes between Days 9 and 14 .

There were no serious adverse events (SAEs) reported during the product trial. A total of ten mild and one moderate adverse events (AEs) were reported by 11 (14\%) subjects. reported usual brand cigarette smoking and myblu use ad libitum from Day 10 of the Study) participants (arm $2 \mathrm{~K})(n=12)$; blue bars represent participants who continued to exclusively using their selected myblu products ad libitum from Day 10 of the Study (arm 2I) $(n=11)$. Definitions of abbreviated BoE can be found in Table 3 (color figure online)

The most frequently reported event was headache, reported by four (10\%) subjects. The principal investigators (PIs) considered all events to be unrelated to study product. During Part 1 of the study, a total of 35 mild and three moderate AEs were reported by 27 (34\%) subjects across study products. The most frequent event was headache, experienced by six ( $8 \%$ ) subjects. All remaining events were reported by four or fewer $(\leq 5 \%)$ subjects each. The PIs considered two events \{throat tightness [myblu ${ }^{\mathrm{TM}}$ Intense Fresh Melon 2.5\%] and oropharyngeal discomfort $\left[\mathrm{myblu}^{\mathrm{TM}}\right.$ Gold Leaf $\left.\left.2.4 \%\right]\right\}$ to be likely related to study product and one event \{headache $\left[m y b^{~}{ }^{\mathrm{TM}}\right.$ Intense Fresh Mint $\left.\left.2.4 \%\right]\right\}$ to be possibly related. All remaining events were considered to be unlikely related/ unrelated to study product. During Part 2, a total of ten mild and two moderate AEs were reported by nine (11\% of total) subjects. The AEs were experienced by four (18\%) subjects in the exclusive smoking of usual brand combustible cigarettes group, three $(12 \%)$ in the exclusive $m y$ blu ${ }^{\mathrm{TM}}$ ENDS use group in Study 2, and two (8\%) in the Study 1 dual use of $m y \mathrm{blu}^{\mathrm{TM}}$ ENDS and usual brand combustible cigarettes 
group. Headache was the most frequently reported event in Part 2, experienced by six (8\%) subjects. All remaining events were reported by one (1\%) subject each. The PI considered all events in Part 2 unlikely related/unrelated to study product. Full breakdown tables of AEs can be found in the supplementary information (Tables 14-17).

\section{Discussion}

\section{BoE levels to selected HPHCs were substantially reduced after switching to exclusive myblu ENDS use}

The first part of this study demonstrated substantial reductions in 14 non-nicotine tobacco smoking-related $\mathrm{BoE}$ in adult smokers following 9 days of exclusive use of $m y$ blu ENDS products across two identical, open label, randomised Studies (1 and 2). Further to this, within the exclusive myblu ENDS use arm of Part 2 of the study, 14 days after first switching, these substantial reductions were maintained. Our findings, that switching from combustible cigarette smoking to exclusive fourth generation ENDS use results in significant and rapid reductions in adult smokers' tobacco smoking-related BoE, are consistent with previous reports, both from clinical settings and at a population level [18, 54-56]. Our studies extend upon previous evidence, for example, we used newer (fourth) generation myblu ENDS products than in the study by O'Connell et al. [22, 26]. It has been postulated that newer generation products have been associated with increased BoE of toxicants in users [57], however, our study reported substantial decreases across a comprehensive selection of smoking-related BoEs to HPHCs following exclusive myblu ENDS use. Furthermore, the comprehensive nature of the BoE assessed provides a higher degree of confidence that adult smokers who transition to exclusive ENDS use will experience reduced exposure to HPHCs over a short period of time. Our study also assessed a larger number of product variants, from one commercial brand, than previous assessments [17, 22]. These products included a larger selection of flavours, nicotine strengths and two different nicotine formulations, and even when combined within study groups, the reductions in combustible tobacco smoking-associated toxicants can still be demonstrated to be substantial following exclusive myblu ENDS use.

The reductions observed in tobacco smoking-related HPHC BoE in this study are perhaps unsurprising as studies on ENDS aerosol chemistry not only provide evidence of reduced HPHC levels, and fewer of these chemicals, in the aerosol itself compared to cigarette smoke $[9,11,12]$, but this also correlates with the reduced, or removed, biological effects within toxicological evaluations [8, 38, 58] and in clinical settings $[17,20]$. If toxicants are reduced, or even absent, in the ENDS aerosol, user BoE will reflect this directly; this could suggest a re-evaluation on the need for tobacco smoking-related BoE clinical studies for ENDS products where aerosol chemistry studies have fully characterised the aerosol. Our findings add to the weight of evidence that exclusive ENDS use results in the reduction of adult smokers' exposure to cigarette-associated toxicants and carcinogens and can have an important role in THR.

\section{Switching to combustible cigarette use reversed reductions in BoE observed after exclusive myblu ENDS use}

Upon randomised adult smoker switching to exclusive combustible cigarette use in Part 2 of the study, the substantial reductions in BoE measured on Day 9 against baseline were reversed by Day 14. This is consistent with the large numbers of HPHCs found in combustible cigarette smoke $[11,12]$, to which users will subsequently be exposed. In both Studies, some increases in BoE were observed compared to baseline on Day 14 in the exclusive combustible cigarette smoking participants. Due to the clinical confinement within the study, participants may have increased their product usage compared to baseline due to boredom or lack of usual distractions, as observed in the study by Jay et al. [17]. The reversal of reductions in BoE seen in the participants between Days 9 and 14 indicates that ENDS users will only experience maximal reductions in exposure to harmful tobacco smoking-related compounds upon complete cessation of cigarette smoking. The findings from the dual-use arm of this study reinforce this further.

\section{Dual use of combustible cigarettes and myblu ENDS may have a role in off-ramping from smoking}

An important part of THR, through providing alternative, reduced harm, nicotine delivery products, is the off-ramping process of adult combustible cigarette smokers from cigarettes to ENDS, for example. This transitional process typically involves a period in which adult smokers may exercise dual use of combustible cigarettes and ENDS while they familiarise themselves with the new products in terms of usage and formulation preferences. Previous studies have found $\mathrm{BoE}$ levels correlated with the level of use of products: Goniewicz et al. [57] documented that higher BoE concentrations in dual-user population samples correlated with greater cigarette smoking frequency and O'Connell et al. [22] found that reductions in BoE levels were generally proportional to the reduction in cigarettes smoked. To our knowledge, there is limited information in the literature on how a period of such dual use of combustible cigarettes and newer generation ENDS affects the BoE levels found in adult smokers. We, therefore, included the dual-use arm in 
Part 2 of this study to investigate this further. As ENDS use is intended as a substitute, and not a supplement, for cigarette smoking, the arm involved reduction of cigarette use to around $50 \%$ of that reported at baseline alongside ad libitum use of the myblu ENDS products available.

Following dual use of cigarettes and myblu ENDS products, where cigarette smoking is reduced, significant reductions were maintained in the majority of smoking-related HPHC BoE compared to baseline cigarette use. This demonstrates the harm reduction potential of substituting cigarette use with ENDS use, even if only to 50\% as a starting point. As expected, these reductions were not to the level of continued exclusive myblu use, indicating combustible cigarettes alone lead to increased HPHC exposure. In fact, dual use in Part 2 of the study still brought some BoE levels to statistically similar levels as the exclusive cigarette groups (in Study 1: NNN; in Study 2: NNAL, HEMA). This highlights the importance of complete smoking cessation following a period of dual use, as exclusive myblu ENDS use in this study appears to offer the greatest degree of harm reduction potential through the most reduced exposure to tobacco smoking-related BoE. However, and as may be expected, the majority of HPHC BoE levels were recorded to be reduced to around $50 \%$ of that seen in the exclusive myblu ENDS use arms, indicating reductions in $\mathrm{BoE}$ are generally proportional to the reductions in cigarette smoking as observed previously [17, 18, 22, 57]. Due to sample size, only one dual use ratio (50\% self-reported daily cigarette consumption plus ad libitum ENDS use) group was included in this study as placing the subjects into smaller groups would reduce the statistical power of the study. However, in the future, it would be interesting to investigate additional patterns of dual usage.

In the dual-use groups, there were some differences observed between the average levels of reduction of specific BoE between Studies 1 and 2, for example, NNN and MHBMA. This may be attributed to the differences in the (majority) nicotine formulation between the two Studies, or may be attributed to individual baseline levels. However this would require further elucidation, perhaps on a product-byproduct basis.

\section{Study products}

Of note within this study is the absence of statistical differences between the levels of nicotine equivalents from baseline both at Day 9 and in any of the groups at Day 14. This is expected as users were allowed to use their products ad libitum for the majority of the study, to achieve their desired level of nicotine satisfaction. This also supports the reduced harm potential of ENDS use: preferred nicotine delivery can be achieved in adult smokers, but with substantially reduced levels of several tobacco smoking-related BoE compared to the cigarette smoking baseline.

The selection of products used in this study contained two different nicotine formulations, nicotine salt (nicotine lactate) and freebase nicotine. Nicotine is commonly present in e-liquids in the freebase form, which, due to its higher volatility than nicotine salts, is expected to be generally absorbed in the upper respiratory tract, and reportedly leads to a harsh, bitter sensory experience for the adult smoker [27]. The more recent innovation of using nicotine salts in the e-liquid formulation, which are formed by the reaction of nicotine with a suitable acid, allows improved delivery to, and, therefore, absorption in, the alveoli, and, therefore, increased plasma nicotine levels [22]. This has been shown to provide greater satisfaction for the adult smoker and, therefore, may encourage their use of ENDS as a combustible cigarette smoking alternative [28]. In this study, seven freebase nicotine products and nine nicotine salt products were tested, and these products were generally allocated to Study 1 or 2 dependent on the type of nicotine within their e-liquid formulation. To ensure an even distribution of products between Studies 1 and 2, to allow the product randomisation schedule to be implemented, one nicotine salt product was allocated to Study 2 and was selected for its comparable nicotine strength to the freebase products within that Study. In both Studies 1 and 2, nicotine levels were not significantly changed from their baseline at Days 9 or 14 in the exclusive myblu ENDS users. However, nicotine equivalents measured in Study 1 participants (nicotine salt products) were higher compared to in the Study 2 participants (mainly freebase nicotine products), which may be expected given the higher nicotine strengths and greater delivery associated with nicotine salt-containing products $[28,59]$. This may also explain the significant difference in nicotine equivalent levels achieved following dual use of combustible cigarettes and the freebase products and exclusive combustible cigarette use. It is also of note, when participants were allowed to use additional products to those they had been allocated in Part 1 of the study, there were slight increases in participants' nicotine equivalent levels, particularly in the freebase nicotine product users (Study 2). This perhaps reinforces the importance of providing consumers with a range of products, to satisfy their nicotine usage preferences, but could also indicate that participants were becoming more familiar with myblu ENDS product usage over the 14 day period of the study.

Due to the large numbers of products used within this study, containing a selection of nicotine strengths, one of two different nicotine formulations and different flavours, it was not possible to analyse formulation-specific BoE. However, the results clearly show, even with a combination of different myblu ENDS products, there were significant reductions in the $14 \mathrm{HPHC} \mathrm{BoE}$ compared to combustible cigarette baseline levels over a short period of time. The 
study was designed to allow participants to use a combination of products. This is consistent with the belief that THR can be maximised by providing a broad portfolio of flavours in combination with multiple nicotine strengths to allow adult smokers to find their preferred product(s) as an alternative for smoking combustible cigarettes. A further strength of this aspect of the study design is that it is also reflective of real world exposure to the ENDS category with the many options of combination of different e-liquid formulations. Additionally, the participants were not experienced ENDS users, therefore, it was important to provide them with these choices; in Part 2 of the study participants were also provided with the additional option to choose alternative products to those they had been randomised to in Part 1 .

\section{Study limitations}

The present study has a number of limitations and the data should be viewed in this context. While the present study demonstrated reductions on BoE following switching of adult smokers to myblu product use, the study did not include an abstinence arm for comparison due to the scale of the wider study. However, similar studies [17, 22] have demonstrated that ENDS use can reduce BoE levels comparable to abstinence itself. The wider study carried out at this time included other analyses including PK and subjective evaluations; these data will be addressed in subsequent publications. If the number of available study arms had permitted, it would have been interesting to compare the results against an appropriate similar product on the market.

The data were not adjusted for multiple comparisons, however, a correction such as Bonferroni could be useful to avoid any false positive outcomes. Application of this correction may have some influence on the outcomes of the study. However, as there were a limited number of groups between which comparisons were being made, this data correction was not included in the objectives of this study. It would be interesting to incorporate this into the analysis of future studies where appropriate.

Upon comparison of the data from the two Studies, baseline BoE levels were generally lower in Study 2 than in Study 1 . This is likely due to variability between the sites and also between the subjects on the recruitment databases at each site, e.g., differences in number of combustible cigarette usage per day, however, this was not analysed formally. Additionally, although reductions were observed in NNN levels in Part 2 of the study in the exclusive myblu use arm, there was a lack of significance between the arms in some cases (between all arms in Study 1; between I and K in Study 2) which may be attributed to the large variability observed in these values within the subject groups.

Although the study did contain a selection of BoE representing major classes of HPHCs associated with smoking-related disease, the study did not include metals, which have been observed to be present in some e-liquids and are of toxicological interest [60]. Further studies may be required to assess the levels of exposure to metals from ENDS usage. However, the main focus of this study was on the BoE from the FDA HPHC list for tobacco products, on which metals are not listed as hazardous or potentially hazardous constituents [37].

Overall, the data we have presented here adds to the weight of evidence that ENDS can contribute to THR by demonstrating substantial reductions in tobacco smokingrelated HPHC BoE in adult smokers following switching to exclusive myblu use over a short period of time. Furthermore, dual use of myblu ENDS and combustible cigarettes, involving cigarette substitution and representative of a period of off-ramping from tobacco smoking, also leads to reductions in HPHC BoE, but not to the same extent as exclusive ENDS use. The study also demonstrated that the adult smoker participants did not experience any SAEs upon use of any of the myblu products, which were deemed to have good safety and tolerability profiles over the 14 days of the study. Future studies, for example into the longer term effects on BoE and tracking biomarkers of potential harm in myblu ENDS users, will further inform on the products' THR potential.

Supplementary Information The online version contains supplementary material available at https://doi.org/10.1007/s11739-021-02813-w.

Acknowledgements The authors thank Celerion, who conducted the commissioned study and analysed the data. Thank you to Imperial Brands' scientific teams and Reading Committee for their critical review of the manuscript and support across the wider project encompassing this work.

Funding This work was funded entirely by Fontem US LLC, a subsidiary of Imperial Brands PLC.

Data availability Study 1 (CA22749) has been registered at www.clini caltrials.gov with ID: NCT 04430634. Study 2 (CA22736) study was also registered at www.clinicaltrials.gov with ID: NCT 04429932.

\section{Declarations}

Conflict of interest This work was funded by Fontem US LLC, a subsidiary of Imperial Brands PLC, and manufacturers of the myblu ${ }^{\text {TM }}$ products used in this study. This work was performed by Imperial Brands PLC on behalf of Fontem US LLC as a service provider. Work was contracted to Celerion, who conducted the study and analysed the data. At the time of the study and/or writing, PM, SM, FC, TV, XC, MS, JT, NC and GOC were employees of Imperial Brands PLC.

Ethical approval The study was carried out in accordance with the ethical principles set forth in the Declaration of Helsinki. The study protocol for Study 1 was reviewed and approved by Advarra Institutional Review Board (IRB) (Columbia, MD) and the study protocol for Study 2 was reviewed and approved by IntegReview IRB (Austin, 
TX). Any subsequent amendments were also approved by the same respective IRBs.

Participant consent All subjects provided informed consent. All prospective subjects had the study explained to them by the investigator or their designee and were required to read, sign and date an Institutional Review Board (IRB) approved Informed Consent Form (ICF) prior to completion of screening or other study procedures.

Open Access This article is licensed under a Creative Commons Attribution 4.0 International License, which permits use, sharing, adaptation, distribution and reproduction in any medium or format, as long as you give appropriate credit to the original author(s) and the source, provide a link to the Creative Commons licence, and indicate if changes were made. The images or other third party material in this article are included in the article's Creative Commons licence, unless indicated otherwise in a credit line to the material. If material is not included in the article's Creative Commons licence and your intended use is not permitted by statutory regulation or exceeds the permitted use, you will need to obtain permission directly from the copyright holder. To view a copy of this licence, visit http://creativecommons.org/licenses/by/4.0/.

\section{References}

1. Royal College of Physicians (2016) Nicotine without smoke: tobacco harm reduction. https://www.rcplondon.ac.uk/proje cts/outputs/nicotine-without-smoke-tobacco-harm-reduction-0. Accessed 6 Aug 2021

2. Polosa R et al (2019) The effect of e-cigarette aerosol emissions on respiratory health: a narrative review. Expert Rev Respir Med 13(9):899-915. https://doi.org/10.1080/17476348.2019.1649146

3. McNeill A, Brose LS, Calder R, Bauld L, Robson D (2018) Evidence review of ENDS and heated tobacco products 2018. A report commissioned by Public Health England, London

4. Polosa R et al (2013) A fresh look at tobacco harm reduction: the case for the electronic cigarette. Harm Reduct J 10(1):19. https:// doi.org/10.1186/1477-7517-10-19

5. Hartmann-Boyce J et al (2020) Electronic cigarettes for smoking cessation. Cochrane Database of Systematic Rev. https://doi.org/ 10.1002/14651858.CD010216.pub4

6. George $J$ et al (2019) Cardiovascular effects of switching from tobacco cigarettes to electronic cigarettes. J Am Coll Cardiol 74(25):3112-3120. https://doi.org/10.1016/j.jacc.2019.09.067

7. Polosa $\mathrm{R}$ et al (2020) COPD smokers who switched to ENDS: health outcomes at 5-year follow up. Ther Adv Chronic Dis 11:2040622320961617. https://doi.org/10.1177/2040622320961617

8. Rudd $\mathrm{K}$ et al (2020) Chemical composition and in vitro toxicity profile of a pod-based ENDS aerosol compared to cigarette smoke. Appl In Vitro Toxicol. https://doi.org/10.1089/aivt.2019.0015

9. Goniewicz ML et al (2014) Levels of selected carcinogens and toxicants in vapour from electronic cigarettes. Tob Control 23:133-139. https://doi.org/10.1136/tobaccocontrol-2012-050859

10. Hajek P et al (2014) Electronic cigarettes: review of use, content, safety, effects on smokers and potential for harm and benefit. Addiction 109:1801-1810. https://doi.org/10.1111/add.12659

11. Tayyarah R, Long GA (2014) Comparison of select analytes in aerosol from ENDS with smoke from conventional cigarettes and with ambient air. Regul Toxicol Pharmacol 70(3):704-710. https://doi.org/10.1016/j.yrtph.2014.10.010

12. Margham J et al (2016) Chemical composition of aerosol from an ENDS: a quantitative comparison with cigarette smoke. Chem Res Toxicol 29(10):1662-1678. https://doi.org/10.1021/acs.chemr estox.6b00188
13. Wieczorek R et al (2020) A comparative in vitro toxicity assessment of electronic vaping product e-liquids and aerosols with tobacco cigarette smoke. Toxicol In Vitro 66:104866. https://doi. org/10.1016/j.tiv.2020.104866

14. Czekala L et al (2019) Toxicological comparison of cigarette smoke and ENDS aerosol using a 3D in vitro human respiratory model. Regul Toxicol Pharmacol 103:314-324. https://doi.org/ 10.1016/j.yrtph.2019.01.036

15. Haswell LE et al (2017) Reduced biological effect of ENDS aerosol compared to cigarette smoke evaluated in vitro using normalized nicotine dose and RNA-seq-based toxicogenomics. Sci Rep 7(1):888. https://doi.org/10.1038/s41598-017-00852-y

16. Breheny $D$ et al (2020) The in vitro assessment of a novel vaping technology. Toxicol Rep 7:1145-1156. https://doi.org/10.1016/j. toxrep.2020.08.016

17. Jay J et al (2019) Five-day changes in biomarkers of exposure among adult smokers after completely switching from combustible cigarettes to a nicotine-salt pod system. Nicotine Tob Res 22(8):1285-1293. https://doi.org/10.1093/ntr/ntz206

18. Hatsukami DK et al (2020) A randomized clinical trial examining the effects of instructions for electronic cigarette use on smokingrelated behaviors and biomarkers of exposure. Nicotine Tob Res 22(9):1524-1532. https://doi.org/10.1093/ntr/ntz233

19. Smith DM et al (2020) Differences in exposure to nicotine, tobaccospecific nitrosamines, and volatile organic compounds among electronic cigarette users, tobacco smokers, and dual users from three countries. Toxics. https://doi.org/10.3390/toxics8040088

20. Akiyama Y, Sherwood N (2021) Systematic review of biomarker findings from clinical studies of electronic cigarettes and heated tobacco products. Toxicol Rep 8:282-294. https://doi.org/10. 1016/j.toxrep.2021.01.014

21. Goniewicz ML et al (2017) Exposure to nicotine and selected toxicants in cigarette smokers who switched to electronic cigarettes: a longitudinal within-subjects observational study. Nicotine Tob Res 19(2):160-167. https://doi.org/10.1093/ntr/ntw160

22. O'Connell G et al (2016) Reductions in biomarkers of exposure (BoE) to harmful or potentially harmful constituents (HPHCs) following partial or complete substitution of cigarettes with electronic cigarettes in adult smokers. Toxicol Mech Methods 26(6):443-454. https://doi.org/10.1080/15376516.2016.1196282

23. Round EK et al (2019) Biomarkers of tobacco exposure decrease after smokers switch to an ENDS or nicotine gum. Nicotine Tob Res 21(9):1239-1247. https://doi.org/10.1093/ntr/nty 140

24. Erythropel HC et al (2020) Differences in flavourant levels and synthetic coolant use between USA, EU and Canadian Juul products. Tob Control 30:453-455

25. Mallock N et al (2020) Trendy e-cigarettes enter Europe: chemical characterization of JUUL pods and its aerosols. Arch Toxicol 94(6):1985-1994

26. CDC. ENDS or vaping products visual dictionary. https://www. cdc.gov/tobacco/basic_information/ENDS/pdfs/ecigarette-or-vaping-products-visual-dictionary-508.pdf. Last accessed 04 Jan 2021

27. Leventhal AM et al (2021) Effect of exposure to ENDS with salt vs free-base nicotine on the appeal and sensory experience of vaping: a randomized clinical trial. JAMA Netw Open 4(1):e2032757-e2032757. https://doi.org/10.1001/jamanetwor kopen.2020.32757

28. O'Connell G et al (2019) A randomised, open-label, crossover clinical study to evaluate the pharmacokinetic profiles of cigarettes and ENDS with nicotine salt formulations in US adult smokers. Intern Emerg Med 14(6):853-861. https://doi.org/10. 1007/s11739-019-02025-3

29. Russell C et al (2018) Changing patterns of first e-cigarette flavor used and current flavors used by 20,836 adult frequent e-cigarette users in the USA. Harm Reduct J 15(1):33. https://doi.org/10. 1186/s12954-018-0238-6 
30. Gravely $\mathrm{S}$ et al (2020) The association of ENDS flavors with satisfaction, enjoyment, and trying to quit or stay abstinent from smoking among regular adult vapers from Canada and the United States: findings from the 2018 ITC four country smoking and vaping survey. Nicotine Tob Res 22(10):1831-1841. https://doi. org/10.1093/ntr/ntaa095

31. Robertson L et al (2019) Dual use of electronic nicotine delivery systems (ENDS) and smoked tobacco: a qualitative analysis. Tob Control 28:13-19. https://doi.org/10.1136/tobaccocon trol-2017-054070

32. Simonavicius E et al (2017) What factors are associated with current smokers using or stopping ENDS use? Drug Alcohol Depend 173:139-143. https://doi.org/10.1016/j.drugalcdep.2017.01.002

33. Zhuang Y-L et al (2016) Long-term ENDS use and smoking cessation: a longitudinal study with US population. Tob Control 25:190 i95. https://doi.org/10.1136/tobaccocontrol-2016-053096 (Suppl 1)

34. Czoli CD et al (2019) Biomarkers of exposure among "dual users" of tobacco cigarettes and electronic cigarettes in Canada. Nicotine Tob Res 21(9):1259-1266. https://doi.org/10.1093/ntr/nty174

35. National Academies of Sciences, Engineering, and Medicine (NASEM); Health and Medicine Division; Board on Population Health and Public Health Practice; Committee on the Review of the Health Effects of Electronic Nicotine Delivery Systems; Eaton DL, Kwan LY, Stratton K (eds) (2018) Public Health Consequences of ENDS. Washington (DC): National Academies Press (US). The National Academies of SCIENCES ENGINEERING MEDICINE. Available from: https://www.ncbi.nlm.nih.gov/ books/NBK507188/. Accessed 6 Aug 2021

36. Kaur $\mathrm{G}$ et al (2018) Immunological and toxicological risk assessment of ENDS. Eur Respir Rev 27(147):170119. https://doi.org/ 10.1183/16000617.0119-2017

37. FDA (2012) Harmful and potentially harmful constituents in tobacco products and tobacco smoke; established list US department of health and human services. Federal Register - The Daily Journal of the United States Government 77(64):20034-20037

38. Czekala $L$ et al (2021) The in vitro ToxTracker and Aneugen Clastogen Evaluation extension assay as a tool in the assessment of relative genotoxic potential of e-liquids and their aerosols. Mutagenesis 36(2):129-142. https://doi.org/10.1093/mutage/geaa033

39. Hatsukami DK et al (2009) Clinical trials methods for evaluation of potential reduced exposure products. Cancer Epidemiol Biomark Prev 18(12):3143-3195

40. Caponnetto P et al (2020) Non-inferiority trial comparing cigarette consumption, adoption rates, acceptability, tolerability, and tobacco harm reduction potential in smokers switching to heated tobacco products or electronic cigarettes: study protocol for a randomized controlled trial. Contemporary Clin Tr Commun 17:100518-100518

41. Gale $\mathrm{N}$ et al (2018) Changes in biomarkers of exposure on switching from a conventional cigarette to tobacco heating products: a randomized, controlled study in healthy Japanese subjects. Nicotine Tob Res 21(9):1220-1227. https://doi.org/10.1093/ntr/nty104

42. FDA (2019) Premarket tobacco product applications for electronic nicotine delivery systems (ENDS). Docket number: FDA-2015-D-2496. https://www.fda.gov/regulatory-information/search-fda-guidancedocuments/premarket-tobacco-product-applications-electronic-nicot ine-delivery-systems-ends. Last accessed $11 \mathrm{Feb} 2021$

43. ICH (2016). E6 (R2) Good clinical practice. https://www.ema. europa.eu/en/ich-e6-r2-good-clinical-practice Last accessed 11 Feb 2021

44. Benowitz NL et al (2009) Nicotine chemistry, metabolism, kinetics and biomarkers. Handb Exp Pharmacol 192:29-60

45. Carmella SG et al (2009) Effects of smoking cessation on eight urinary tobacco carcinogen and toxicant biomarkers. Chem Res Toxicol 22(4):734-741. https://doi.org/10.1021/tx800479s
46. Gregg EO et al (2013) Urinary biomarkers of smokers' exposure to tobacco smoke constituents in tobacco products assessment: a fit for purpose approach. Biomarkers 18(6):467-486. https://doi. org/10.3109/1354750X.2013.821523

47. Hecht SS (2002) Human urinary carcinogen metabolites: biomarkers for investigating tobacco and cancer. Carcinogenesis 23(6):907-922. https://doi.org/10.1093/carcin/23.6.907

48. Hecht SS et al (2010) Applying tobacco carcinogen and toxicant biomarkers in product regulation and cancer prevention. Chem Res Toxicol 23(6):1001-1008. https://doi.org/10.1021/tx100056m

49. US Department of Health and Human Services (2014) The health consequences of smoking-50 years of progress: a report of the surgeon general. Atlanta, GA US Department of Health and Human Services, Centers for Disease Control and Prevention, National Center for Chronic Disease Prevention and Health Promotion, Office on Smoking and Health

50. Yuki D et al (2018) Assessment of the exposure to harmful and potentially harmful constituents in healthy Japanese smokers using a novel tobacco vapor product compared with conventional cigarettes and smoking abstinence. Regul Toxicol Pharmacol 96:127-134. https://doi.org/10.1016/j.yrtph.2018.05.001

51. FDA (2013) Bioanalytical method validation guidance for industry. Docket number FDA-2013-D-1020. https://www.fda.gov/regul atory-information/search-fda-guidance-documents/bioanalyticalmethod-validation-guidance-industry. Last accessed 12 Feb 2021

52. FDA (2020) 21 CFR part 58 good laboratory practice for nonclinical laboratory studies. https://www.gmp-compliance.org/files/guide mgr/CFR-2020-title21-vol1-part58.pdf. Last accessed 12 Feb 2021

53. EMA (2009) Guideline on bioanalytical method validation. https:// www.ema.europa.eu/en/documents/scientific-guideline/guidelinebioanalytical-method-validation_en.pdf. Last accessed 12 Feb 2021

54. D'Ruiz CD et al (2016) Reductions in biomarkers of exposure, impacts on smoking urge and assessment of product use and tolerability in adult smokers following partial or complete substitution of cigarettes with electronic cigarettes. BMC Public Health 16:543-543. https://doi.org/10.1186/s12889-016-3236-1

55. Shahab L et al (2017) Nicotine, carcinogen, and toxin exposure in long-term ENDS and nicotine replacement therapy users: a cross-sectional study. Ann Intern Med 166(6):390-400. https:// doi.org/10.7326/M16-1107

56. McRobbie $\mathrm{H}$ et al (2015) Effects of switching to electronic cigarettes with and without concurrent smoking on exposure to nicotine, carbon monoxide, and acrolein. Cancer Prev Res (Phila) 8(9):873-878. https://doi.org/10.1158/1940-6207.CAPR-15-0058

57. Goniewicz ML et al (2018) Comparison of nicotine and toxicant exposure in users of electronic cigarettes and combustible cigarettes. JAMA Netw Open 1(8):e185937-e185937. https://doi.org/ 10.1001/jamanetworkopen.2018.5937

58. Simms L et al (2020) The use of human induced pluripotent stem cells to screen for developmental toxicity potential indicates reduced potential for non-combusted products, when compared to cigarettes. Curr Res Toxicol 1:161-173. https://doi.org/10.1016/j. crtox.2020.11.001

59. Yingst JM et al (2019) Nicotine absorption profile among regular users of a pod-based electronic nicotine delivery system. JAMA Netw Open 2(11):e1915494-e1915494. https://doi.org/10.1001/ jamanetworkopen.2019.15494

60. Sakamaki-Ching S et al (2020) Correlation between biomarkers of exposure, effect and potential harm in the urine of electronic cigarette users. BMJ Open Respir Res 7(1):e000452. https://doi. org/10.1136/bmjresp-2019-000452

Publisher's Note Springer Nature remains neutral with regard to jurisdictional claims in published maps and institutional affiliations. 